\title{
Virtual Reality Tool for Rehabilitation of Patients with Parkinson's Disease: A Conceptual Design Review
}

\author{
Josiah Cyrus BOQUE, Jaime CARO, Richelle Anne JUAYONG and \\ Roland Dominic JAMORA \\ Department of Computer Science, \\ College of Engineering, \\ University of the Philippines - Diliman
}

\begin{abstract}
Parkinson's Disease is a progressive, irreversible disease that is only slowed down with the use of medications and therapy. These are the only way to slow down the progression of the disease to more severe situation. Currently, virtual reality is being use on games and entertainment. Some doctors prefer the use of virtual reality like Wii to help supplement the therapy done on rehabilitation. However, devices to cater virtual reality for mid-class to lower-class patients cannot afford such devices. An alternative device, usually accessible to everyone is proposed to cater these virtual reality applications that can help in therapy of Parkinson's patients. With that, the capabilities of VR can be accessible to more patients who cannot avail expense medication and devices for virtual reality.
\end{abstract}

Keywords. Virtual Reality, Parkinsons Disease, VR Rehabilitation

\section{Introduction}

In recent years, mental health and disorder has become a concern all over the world and is increasing. Instances of imbalances and involuntary seizures may show different symptoms enough for someone to be diagnosed with this disorder. One of these is Parkinson's Disease (PD). Up to this date, it is irreversible. However, the progression of symptoms can be slow down, and let the disease stop prevailing at the early stages through medication and continuous therapy[1].

There hasn't been a focus of research to this disease on slow-developing country like in the Philippines. Moreover, ratio of doctors and scientists that studies for treatment and core medication per population is relatively small. Hence, patients with PD endure on having the disease as they age, then eventually die bed-ridden [2]. Moreover, PD can be acquired at any age, but as they age, incidence of PD symptoms are also imminent. Risk of a person to have PD can be high due to different factors like race, demography, environmental noise and pollution, and others [1].

On this paper, problems on available treatment will be addressed, then a design of a proposed alternative solution using other means of Virtual Reality (VR) technology will be created to supplement and help catalyze the therapy treatment process done on PD patients. Hence, to help slow down the progression of symptoms in patients. 


\section{Review of Related Literature}

\subsection{Parkinson's Disease}

Parkinson's disease (PD) is a nervous system disorder where the nerve cells that produces dopamine are being affected. Hence, signs of this disease can be early depicted by the following symptoms: muscle rigidity, tremors, and changes in speech and gait (walking posture). A part of your brain called the substantia nigra makes our dopamine, a chemical gives way for signals from neurons to our brain, especially when we think and do something. This is also the chemical that manages how smooth and evenly we move. But having this disorder, we are tend to become disoriented on how we navigate, do our activities or even on the way we talk [3].

PD severity can be measured via the Hoehn and Yahr staging of PD based on the study of Hoehn and Yahr that focused on factors within and around a person that may have a significant effect which triggers the disease's severity, or the symptoms patients may show. Some of these factors include age, physical activities, hobbies and etc. And when these factors are identified, the patient undergoes a test that involves certain movement routine [4]. Using this scale, they found out that progression for each patient to jump to a higher stage takes 2-5 years even if patient is on rehabilitation [5]

Balance and posture are usually lost in PD patients. Tests such as the Berg Balance Scale (BBS) are used to assess patient. The test includes scoring of 14 movement items, with 4 being the highest score for each item. Each item is scored based on assessment if the patient cannot do the task or has a struggle, or pain doing it. Once the scores have been summarized, the accumulated points will determine the condition of the patient [6].

In the present, drug medication are also still used as a part of the treatment. Usual prescription of doctors may cost patients too much. Computation of each prescription of doctors to the patients can show that each patient have to spend of at least $\$ 6.00$ just for the medicines, or at an average of $\$ 6,000$ or PhP 300,000 annually. Dosage of medicines may increase depending on how symptoms persist with the patient. [2].

\subsection{Virtual Reality and its use to Parkinson's Therapy}

Virtual Reality(VR) is a computer-based technology that immerses its users to replicated environments, natural or fictional, as if the user is experiencing it for real. Interaction within the virtual environment can be experience depending on what material is being used. This technology brings us to places or let us do activities that we cannot do in real world. Virtual Reality nowadays is becoming prominent among people, since most mobile devices are now equip with gyroscope or gyro sensors which stimulates the phone to feel heights and direction does the phone is facing towards. Some equipments that are used in this technology are: the computer that renders the visual interface of the environment it want to portrait; the output monitor through a projector, a VR goggles, or digital display (Simulated Television, etc.) [7]. 


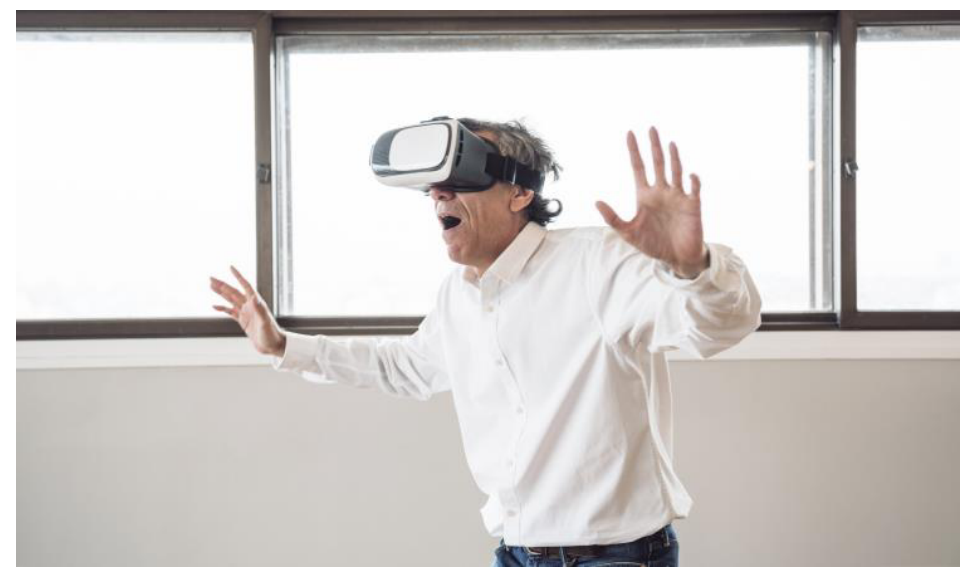

VR as a clinical therapy tool has been found to be effective in improving mobility of patients undergoing such therapy like for cerebral palsy patients. With their 59 participants, improvements with their performance is noticeable as seen in Figure 1.
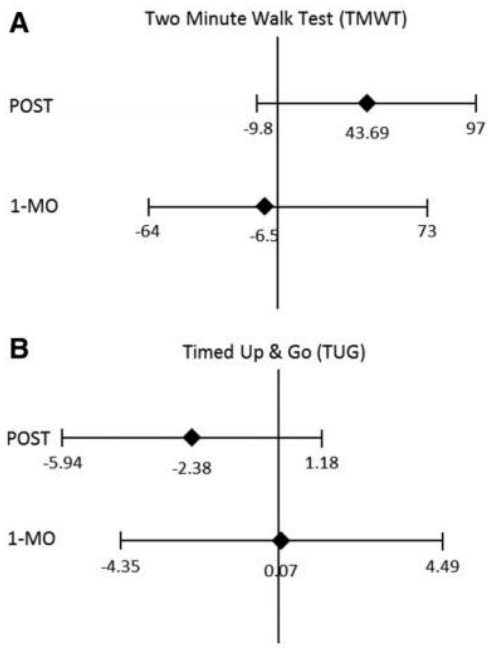

Figure 1. Scores for the Post assessment (POST) and after-a-month assessment (1-MO) for TMWT and TUG

Ninety-five percent confidence intervals and effect size (black diamonds) for the difference in improvements immediately after the final training session (POST) and 1 month after the cessation of training (1 MO) are shown. For the Two-Minute Walk Test (TMWT; A), the effect size to the right of the zero line indicates an improvement, whereas for the Timed Up and Go test (TUG; B), the effect size to the left is indicative of improvement in favor of the treatment group. Moreover, they even did another intervention a month after their post intervention with the patient. They found increase of performance from both of their experimental and controlled group. This only shows that VR therapy has no significant difference with the therapy done on rehabilitation centers. But with the advantage that it can be done at home and by their own means, a lot of patients can now have therapy at their own reach [8]. 
Also, [9] cited the use of Wii Fit, a VR gaming application on Wii, is being use for therapy sessions of stroke patients. Activities included during the intervention involves arms and shoulder movements, flexion and extension of elbow, wrist and finger stretches. With their 22 randomly selected patients, their performance where assessed using the Wolf Motor Function Test. They say that most of their patients have improved significantly in terms of muscle and joint flexes of arms and shoulder. This tells us that VR therapy can really boost patients improvement while enjoying therapy through this kind of technology.

\subsection{Wii Technology}

Wii, a VR technologies, has been used as a tool for Parkinson's Disease therapy with the application called Wii Fit created back 2012. This application is a group of games that are made to train couple of patients' body parts including balance and standing posture test when moving the arms, legs, hips, bending sideways and other extremities.Some of this games include Ski Slalom, Tightrope walk. This is tested with 50 patients, where a study group, 27 of them were able to rehab with the application, while the other 23 , the control group, undergo the conventional therapy. For their preliminary experiment, each patient undergone pre-assessment and post-assessment using BBS and the Dizziness Handicap Inventory (DHI). DHI is a 25 questionnaire-type assessment tool for balance where patients are scored between 4 (affirmative), 2 (sometimes), 0 (negative).
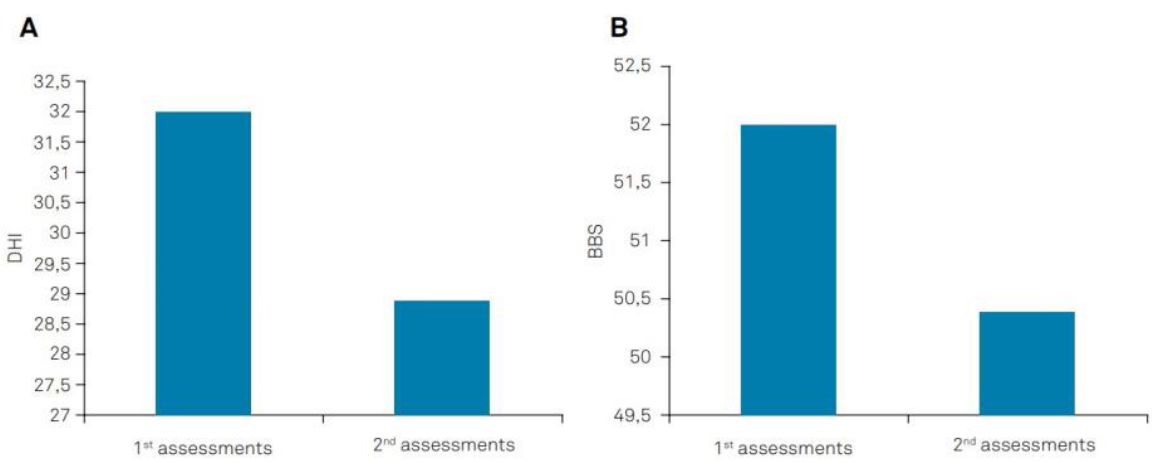

Figure 2. Results of $1^{\text {st }}$ and $2^{\text {nd }}$ Assessment for (A) DHI and (B) BBS.

Based on Figure 2, patients that undergone therapy with Wii Fit shows lowers scores on both DHI and BBS. This indicates that Wii Fit indeed helps to lessen and tremors that patients experience. Moreover, it help the patients improve their body balance and walking posture with reduced fall-risks and increase self-confidence and independence. Test results show that the performance of the study group when performing the games on the Wii Fit were better than that of the control group. This tells us that such use of technology, like the Wii Fit can be a reliable and valid measuring tool to train and assess patients' posture and balance improvements. Moreover, patients experiencing it for the first time, found the technology motivating and exciting to use every time they will do rehabilitation with the technology [10].

Also another paper [11] showed great improvements on patient when they used Wii Fit as part of their therapy session. They created the study as a pilot study to check the significant effect of training when using such gadget. According to the authors, for 10 
weeks, 8 subjects or patients with ages between 30-60 years old underwent their $30 \mathrm{~min}$ sessions per week which includes yoga, balance, aerobic and strength Wii Fit ${ }^{\mathrm{TM}}$ activities. Each activity are also encourage to be modified with increasing difficulty to increase their performance and improvement.

Table 1. Effect of Wii Fit ${ }^{\mathrm{TM}}$ intervention

\begin{tabular}{ccc}
\hline Measurement & Pre-intervention & $\begin{array}{l}\text { Post- } \\
\text { intervention }\end{array}$ \\
\hline 6-min walk test $(\mathrm{m})$ & $650.8( \pm 70.9)$ & $683.6 \pm 85.8)$ \\
$\begin{array}{c}\text { Step test (number) } \\
\text { Right }\end{array}$ & $21.75( \pm 3.4)$ & $22.38( \pm 3.38)$ \\
Left & $22.5( \pm 3.34)$ & $23.13( \pm 3.36)$ \\
Timed Up and Go test(s) & $4.93( \pm 0.76)$ & $5.00( \pm 0.73)$ \\
Timed Up and Go & $5.39( \pm 0.97)$ & $4.94( \pm 0.78)$ \\
cognitive test* & & \\
\hline
\end{tabular}

As seen in Table 1, it shows us the scored they gathered from different test before and after the intervention. They used the 6-mins walk test, Timed Up and Go, Step test and other test as metrics to measure improvement for the patients. Based on the scored, the use of Wii Fit ${ }^{\mathrm{TM}}$ help in improving each patient's strength and balance after training with it. They have seen big improvement on their functional balance measures, flexibility, reaction time or somatosensation.

With these results, the authors are not confident to say that Wii Fit ${ }^{\mathrm{TM}}$ can effectively help patient to have better conditions so they suggest to increase the number of subjects which includes healthy subjects so they can compare how well this technology can go further to improve the performance of the patients as same as with those that are healthy.

\subsection{Motor Imagery}

Moreover, patient assessment using Motor Imagery (MI) together with VR was also studied to improve patients' walking gait, balance and mobility. They collated various articles and papers that indicates improvement on patients with freezing of gait (FOG). These collectively concluded that MI can really help improve patients cognitive and visual learn. And with the help of immersion to VR, patients greatly improve their locomotor movements even more [12].

\subsection{Visuo-motor Learning}

Also, a study showed what with the use of visuo-motor learning through immersive 3D VR can help improve their conditions. They immersed patients from different age group. First they noticed that elders and young subjects have different pace movement. However they are able to observe same result that as they go on with the trials, both age group manage to improve their performance, and lessen movement errors. Second, they found out that PD patients, as compared with their healthy proponents, shows slower comprehension with training using biaxial visuo-motor discordance. Also, they have more errors with the proper movement corrections. However, trends of improvement 
from all subjects shows decreasing amount of movement errors as number of trials are being done.

In general, these applications created for patient rehabilitation are found efficient since these can really help the patients improve their walking gait, balance to gain independence, and can somehow do their daily living activities independently. However, to warrant all claims of improvement when using VR for PD patients' rehabilitation, further studies are still needed to be done. Since there are some proven concepts of improvement, other capacities of VR on brain rehabilitation can now be explored, not just for PD patients' use but also for other neuro-diseases. [13,14].

\section{Proposed Design}

From the previous sections, there are proven concept presented when using VR as therapy for PD patients. However, they are only limited to be used in rehabilitation centers. So to cater patients from places far from these centers, we can create VR applications that can be placed on smartphones and use it as daily therapy for the patients even if they are at staying at their homes.

With Google, they extended the accessibility of VR for making android phones capable of running these applications and programs for a Head-mounted Device (HMD) . With the use of gyroscopes, the phones can manage to sense device orientation, proximity and elevation [15].

Considering the accessibility of android phones to the public that can be a HMD, we can use this as an advantage to make applications for therapy. With that, an application can be created to let its users do the activities similar with what they do in rehabilitation center. If in therapy sessions with Wii Fit, they let the patients bend their body sideways, there can be activities in VR Android phones that will let the users or patients bend their bodies to perform the activities indicated. Also, this application can include games that will test patients' balance, stature strength, and their ability to walk. These are the aspects of the body we can target for their therapy.

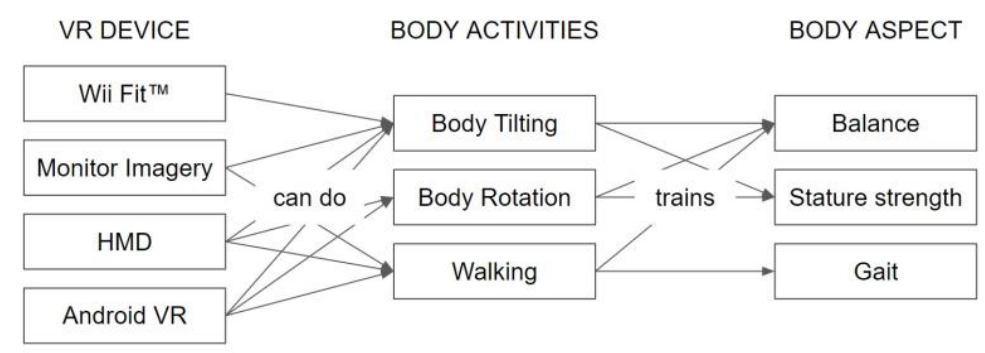

Figure 3. Mapping of activities that can be done with each VR Device. Also the mapping to the body aspects that each activity targets to train

As seen in Figure 3, there are similarities on activities that all VR device can do like from body tilting to actual walking. For Parkinson's Disease, it is very helpful to keep patients moving through walking and limbs movements. These decrease the prevalence of having frozen joints and lessen their gait. So forcing the patients to have fun with VR activities will really be helpful in keeping them moving. Since we target those body parts that other VR devices do too, we can expect that it can also improve the balance, stature strength and gait in training them to walk. 


\subsection{Patient Specification}

For the research, it is recommended to have at least 16 subjects with Parkinson's disease for a pilot test. However there are some limitation about who we will be needed. These patients must :

1. have Parkinson's Disease of stage 2, 2.5, or 3 (according to Hoehn and Yahr Scale);

2. be able to sit and/or stand even if with or without assistance;

3. be able to walk of at least 4 to 6 inches per pace;

4. not have difficulty(no pain) in moving upper(arms, hands, shoulders, etc.) andlower extremities (limbs, feet, legs, etc.).

\subsection{Testing Process}

The testing phase will have two parts. First, after the application is created, a dummy testing will be done to test it for any bugs that a healthy individual can detect or notice while using the current application. After assessing it through a Usability test and Reliability test, the application will then be modified to cater tester's issues with the application, then another testing will be done using the same assessing tests to gather other hanging issues about the application.

Second part of the testing phase involves the participation of random select patients as subjects prior to our set limitations. A pre-assessment will be done to all of them using the application. After which, half of the subjects will undergo rehabilitation with the use of the application for 10 mins. in a span of 4 weeks schedule. Same through with the other other subjects, but they will not be using the application during the testing schedule. After 4 weeks prior to the start of this testing phase, a post-assessment will be done in terms of their performances according to doctor's perspective.

\subsection{Risk and Safety Analysis}

Each of our subjects are assigned a personal assistant(PA) since from the start of their rehabilitation. To be able to successfully apply this testing, coordination with their PA on how to use and guide the subjects with the use of the device and application should be observed. Careful handling of the patient while the testing is on going will dictate safety in using this technology.

\section{Conclusion}

VR has a lot of potential use with its wide variety of activities that can be done and enjoyed according to the literature. Because of the fluidity that the activities that can be ran across different devices, it is surely that application for android phones that will target similar body aspects is also promising. With the proposed use of HMD on smartphones, use of VR for therapy can open accessibility to PD patients living far from rehabilitation centers.

To really test out its capabilities, clinical trials can be done to test the significance of using VR as a supplementary therapy for our patients. Also, Augmented reality (AR) is noticeable nowadays. It it because of its capabilities to place the virtual objects into the real world with the use of mobile devices. This connects the bridge for innovations with AR and VR for health and other life aspects. 


\section{Acknowledgement}

The researchers would like to acknowledge the Engineering Research and Development for Technology under the Department of Science and Technology of the Philippines for funding this research and submission for this conference.

\section{References}

[1] L.C. Tan, Epidemiology of Parkinson's disease, Neurology Asia 2013 (2013), 231-238.

[2] R.D.G. Jamora and J.M. Miyasaki, Treatment gaps in Parkinson's disease care in the Philippines, Neurodegenerative Disease Management 7(4) (2017), 245-251. doi:10.2217/nmt-2017-0014.

[3] Parkinson's Disease: Symptoms, Causes, Diagnosis, Treatment, WebMD, 2017. https://www.webmd.com/parkinsons-disease/parkinsons-disease-overview1.

[4] E. Modestino, Hoehn and Yahr staging of Parkinson's disease in relation to neuropsychological measures, Frontiers In Bioscience, Landmark 23 (2018), 1370-1379. doi:10.2741/4649.

[5] M.M. Hoehn and M.D. Yahr, Parkinsonism: onset, progression, and mortality, Neurology 17(5) (1967), 427-427-. doi:10.1212/wnl.17.5.427.

[6] F.B. Horak, D.M. Wrisley and J. Frank, The Balance Evaluation Systems Test (BESTest) to Differentiate Balance Deficits, Physical Therapy 89(5) (2009), 484-498-. doi:10.2522/ptj.20080071.

[7] Step into a new world of Virtual Reality(VR). https://www.completegate.com/2016070154/blog/virtualreality-explained.

[8] D. Mcewen, A. Taillon-Hobson, M. Bilodeau, H. Sveistrup and H. Finestone, Virtual Reality Exercise Improves Mobility After Stroke, Stroke 45(6) (2014), 1853-1855-. doi:10.1161/strokeaha.114.005362.

[9] V. Fung, A. Ho, J. Shaffer, E. Chung and M. Gomez, Use of Nintendo Wii Fit in the rehabilitation of outpatients following total knee replacement: a preliminary randomized controlled trial, Physiotherapy 98 (2012), 183-188.

[10] J.C. Nitz, S. Kuys, R. Isles and S. Fu, Is the Wii Fit ${ }^{\mathrm{TM}}$ a new-generation tool for improving balance, health and well-being? A pilot study, Climacteric 13(5) (2010), 487-491. doi:10.3109/13697130903395193.

[11] A. Mirelman, I. Maidan and J.E. Deutsch, Virtual reality and motor imagery: Promising tools for assessment and therapy in Parkinsons disease, Movement Disorders 28(11) (2013), 1597-1608-. doi: $10.1002 / \mathrm{mds} .25670$.

[12] K. Dockx, V.V.D. Bergh, E.M. Bekkers, P. Ginis, L. Rochester, J.M. Hausdorff, A. Mirelman and A. Nieuwboer, Virtual reality for rehabilitation in Parkinsons disease, Cochrane Database of Systematic Reviews (2013). doi:10.1002/14651858.cd010760.

[13] N.-Y. Lee, D.-K. Lee and H.-S. Song, Effect of virtual reality dance exercise on the balance, activities of daily living, and depressive disorder status of Parkinson's disease patients, Journal of Physical Therapy Science 27(1) (2015), 145-147-. doi:10.1589/jpts.27.145.

[14] A. Faisal, Computer science: Visionary of virtual reality, Nature 551(7680) (2017), 298-299-. doi:10.1038/551298a. 